\title{
Cutting the chorda tympani: not just a matter of taste
}

\author{
N Guinand*, T Just†, N W Stow $\neq$, H CAO VAN*, B N Landis*§
}

\begin{abstract}
Introduction: Chorda tympani injury as a complication of middle-ear surgery has been extensively studied with regard to its effects upon taste. However, the chorda tympani also carries parasympathetic fibres to the salivary glands of the oral cavity. To date, little has been reported about the effect of chorda tympani section upon salivary function.

Setting: Tertiary care centre.

Material and methods: We report a case series of three patients with bilateral chorda tympani lesions. Chorda tympani function was assessed using 'taste strips' and unstimulated sialometry. A careful history of oral symptoms was taken.

Results: All patients showed transient or permanent bilateral ageusia of the anterior two-thirds of the tongue, and a decreased resting salivary flow rate. In addition, all patients suffered from transient or persistent, distressing xerostomia.

Conclusion: Taste disorders may occur after middle-ear surgery but they are mostly transient, even when the chorda tympani nerves are sectioned bilaterally. In contrast, bilateral chorda tympani lesions may lead to severe, persistent and distressing xerostomia. Based on this neglected aspect of chorda tympani function, we emphasise the importance of preserving the chorda tympani whenever possible.
\end{abstract}

Key words: Chorda Tympani; Taste; Facial Nerve Injuries; Otologic Surgical Procedures

\section{Introduction}

The chorda tympani is considered to be a branch of the facial nerve, and derives all of its fibres from the nervus intermedius. Unlike the facial nerve, the nervus intermedius does not have motor fibres, but contains gustatory fibres from the anterior two-thirds of the tongue, parasympathetic fibres to all the salivary glands (except the parotid glands) and somatosensory branches of the VIIth nerve. Accordingly, chorda tympani injury symptoms resemble those reported after nervus intermedius injury. ${ }^{1}$

The chorda tympani course runs through the middle-ear cavity and is particularly vulnerable to injury by chronic inflammatory processes or middle-ear surgery. ${ }^{2,3}$ The occurrence of taste disorders following middle-ear surgery have been reported for over a century, ${ }^{4}$ and continue to be problematic. ${ }^{5}$ There are still diverse opinions among otologists about what constitutes acceptable manipulation of the chorda tympani during surgery. ${ }^{6-8}$ Based upon their clinical observations, House and Rice both claimed that cutting the chorda tympani, rather than leaving it severely traumatised, resulted in a lower incidence of taste disturbance. ${ }^{9,10}$ While certain authors still defend this point of view, ${ }^{8}$ recent studies have suggested that preserving the chorda tympani is preferable, ${ }^{5,6}$ because long-term recovery of taste function is far better if the chorda tympani remains intact. ${ }^{3}$
Section of the chorda tympani not only affects taste, but also leads to parasympathetic denervation of the ipsilateral submandibular, sublingual and accessory oral salivary glands. Parasympathetic chorda tympani fibres are responsible for the basal secretomotor innervation of these glands; consequently, a diminished salivary flow rate and xerostomia have been reported after middle-ear surgery. ${ }^{1}$

Based on a case series of patients, this paper presents a discussion of the under-reported symptom of xerostomia following bilateral chorda tympani nerve injury.

\section{Methods}

\section{Taste testing}

The gustatory function of the anterior two-thirds of the tongue was tested using 'taste strips', a clinical identification test based on 16 impregnated filter papers. Normal taste function was defined as the correct identification of nine or more taste strips on one side of the tongue (see Landis et al. ${ }^{11}$ for details).

\section{Saliva measurement}

Previous studies have suggested that the subjective feeling of oral dryness is most strongly correlated with unstimulated saliva flow. ${ }^{12}$ Therefore, unstimulated saliva flow

From the *Department of Clinical Neurosciences, Division of Otorhinolaryngology Head and Neck Surgery, University of Geneva Medical School, Switzerland, the $†$ Department of Otorhinolaryngology Head and Neck Surgery, University Hospital of Rostock, Germany, the $\ddagger$ Department of Otorhinolaryngology, Head and Neck Surgery, Mona Vale Hospital, Sydney, New South Wales, Australia, and the §Smell and Taste Clinic, Department of Otorhinolaryngology, Medical School, Technische Universität Dresden, Germany.

Accepted for publication: 14 January 2010. First published online 7 April 2010. 
was measured by placing three sterile gauze pads at the orifices of the submandibular and parotid glands for 6 minutes, in the absence of chewing or salivary stimulation. The pads were weighed before and after this time period. According to previous reports, saliva flow rates below $2 \mathrm{ml}$ per 6 minutes are considered pathologically low. ${ }^{13}$

\section{Results}

\section{Case one}

A 66-year-old woman presented with mouth dryness which had started six weeks after a canal wall up mastoidectomy for a cholesteatoma of the right ear. Interestingly, her mouth dryness disappeared during eating but remained distressing between meals. No xerophthalmia or dry nose was reported. The patient was otherwise well and did not take any medication. She had undergone middle-ear surgery for a left ear cholesteatoma 20 years ago. According to reports from both operations, the chorda tympani had been severed in both ears.

Examination of the patient's taste perception revealed correct identification of three taste strips on the right side and four on the left side (normal value, more than nine). Unstimulated sialometry indicated a total salivary flow rate of $0.5 \mathrm{ml}$ after 6 minutes (normal value, more than $2 \mathrm{ml}$ over 6 minutes). ${ }^{13}$

A labial accessory gland biopsy had been previously undertaken, and revealed normal tissue histology.

Despite trials of artificial saliva and pilocarpine, the patient's symptoms had remained unchanged in the 18 months since her second operation.

\section{Case two}

A 52-year-old woman presented with a history of two uncomplicated operations for otosclerosis of the left ear, performed 20 years ago. Her hearing had improved postoperatively and no dysgeusia had been noted. Surgery had been performed for otosclerosis of the right ear two years ago. Post-operatively, she had complained of taste loss, lingual paraesthesia and mouth dryness. While the lingual paraesthesia had eventually resolved, her taste function and xerostomia had not recovered. No xerophthalmia or dry nose was reported. The patient did not take any medication and was otherwise well. All operation reports were obtained, but none contained any details about intra-operative manipulation of the chorda tympani.

Examination showed bilateral ageusia of the anterior two-thirds of the tongue: five taste strips were detected on the left side and four on the right side (normal value, more than nine). The patient was able to discriminate the four basic tastes only when the substances were given as whole mouth stimuli. Unstimulated sialometry showed a total saliva flow rate of $0.2 \mathrm{ml}$ over 6 minutes (normal value, more than $2 \mathrm{ml}$ over 6 minutes). Treatment with artificial saliva did not improve the patient's complaint.

\section{Case three}

A 35-year-old man had undergone a tympano-atticotomy of the right ear two years ago to treat a small attic cholesteatoma. His hearing had improved post-operatively, but he had developed some ipsilateral dysgeusia in the six months prior to presentation. This dysgeusia was of very short duration and was triggered by touching the right tragus, a phenomenon termed tactile dysgeusia. ${ }^{14}$ According to the operation report, the chorda tympani had been severed. A year later, the patient had undergone a tympano-atticotomy on the left ear, and had suffered subsequent ipsilateral taste loss and xerostomia. According to the operation report, the chorda tympani had been stretched but not sectioned. The patient did not take any medication and was otherwise well.

Taste testing scores were initially six strips on the right side and four on the left side (normal value, more than nine). Unstimulated sialometry showed a total salivary flow rate of $0.3 \mathrm{ml}$ over 6 minutes (normal value, more than $2 \mathrm{ml}$ over 6 minutes).

However, the patient's taste loss and xerostomia recovered slowly over the following months, and had subjectively returned to normal seven months after surgery. Objectively, taste testing showed an unchanged result on the right side but an improved result (10 strips) on the left side. Unstimulated sialometry also improved to almost normal values, with a total salivary flow rate of $1.2 \mathrm{ml}$ after 6 minutes.

\section{Discussion}

In 1965 , Bull ${ }^{1}$ described a series of 25 patients who had undergone bilateral middle-ear surgery, and reported that 16 (65 per cent) suffered from long-lasting and persistent mouth dryness. Furthermore, these patients either carried a bottle of water to sip, or sucked boiled sweets almost continually, in order to alleviate their mouth dryness.

The cases presented in the current paper had all undergone bilateral middle-ear operations separated by an interval of several years. In all cases, both operations had been successful from the hearing point of view, but xerostomia and taste disturbance had developed after the second operation. While the dysgeusia eventually resolved, the xerostomia was persistent and disabling in two patients and transient in the one patient who recovered taste function. Detailed information on whether the chorda tympani nerve had been preserved or sectioned during surgery was not available in all cases.

The sublingual and submandibular salivary glands are responsible for two-thirds of basal saliva production, whereas the parotid glands accounts for less than onethird. Note that this proportion is reversed during meals, when saliva production is stimulated and parotid saliva production increases greatly. Since the parasympathetic fibres innervating the nasal and lacrimal glands leave the nervus intermedius facial nerve at the level of the geniculate ganglion, and do not course through the middle ear with the chorda tympani, nasal and ocular dryness is not reported as a consequence of chorda tympani section.

The literature shows that bilateral section of the chorda tympani performed for the treatment of drooling is effective ${ }^{15}$ while unilateral chorda tympani section does not significantly change saliva flow or subjective xerostomia. ${ }^{6}$ Reduced basal saliva production may lead to mouth dryness, dysphagia, sleep-disordered breathing, dental infection, dysphonia, a sensation of oral burning, or pain. Considering these potential consequences of bilateral chorda tympani section, preservation of the chorda tympani is preferable whenever possible.

In post-operative patients in whom chorda tympani function is in doubt, the repeated assessment of taste function over time may provide useful information. In cases in which the chorda tympani is manipulated but preserved, taste function decreases initially but recovers within a few months, ${ }^{3,5}$ as seen in our third patient. In contrast, a sectioned chorda tympani will not recover to the level of normal function, even if the patient's symptoms resolve. ${ }^{3,17-19}$ In our second patient, taste testing showed bilateral ageusia of the anterior two-thirds of the tongue more than two years after surgery, suggesting bilateral section of the chorda tympani. In our third patient, xerostomia resolved in parallel with recovery of taste function on 
the right side of the tongue, indicating recovery of the chorda tympani nerve, which was probably severely traumatised during surgery.

Furthermore, in patients who have had previous middleear surgery and who require surgery in the opposite ear pre-operative testing of chorda tympani function could inform both patient and surgeon about the risk of postoperative xerostomia and taste disturbance. This testing should assess both resting saliva flow and taste function, and should be performed bilaterally. Intact taste function is a very reliable indicator of intact chorda tympani function; hence, parasympathetic fibres and salivary function are very likely to be intact as well. In contrast, if the chorda tympani has been permanently damaged by the initial surgery, this will be reflected by a pathological taste test result on that side.

- The chorda tympani nerves convey not only taste fibres but also parasympathetic fibres to the ipsilateral sublingual, submandibular and accessory salivary glands of the oral cavity

- Section of one chorda tympani goes mostly unnoticed as regards gustatory and salivary function, because deficits are well compensated by the contralateral nerve

- In contrast, section of both chorda tympani may result in not only gustatory but also salivary symptoms, such as resting xerostomia

- In patients who have had previous middle-ear surgery and who require surgery in the contralateral ear, pre-operative testing of chorda tympani function may inform both patient and surgeon of the risk of post-operative xerostomia and taste disturbance

Unlike early otological reports, ${ }^{9,10}$ which stated that sectioning the chorda tympani produced fewer complications than stretching or manipulating the nerve, recent studies have supported preservation of chorda tympani integrity. 5 There are many reports suggesting that section of the chorda tympani leads to more severe and persistent taste symptoms. $1,3,5,6,20,21$

The dilemma for otological surgeons is not whether to cut or preserve the chorda tympani but, rather, whether to cut or retain severely traumatised chorda tympani nerves. Based on the reported three cases, it is impossible to answer this question. However, subjectively perceived taste symptoms such as dysgeusia disappear regardless of whether the chorda tympani is cut or traumatised. In contrast, almost 60 per cent of patients with sectioned chorda tympani never recover any electrogustometric responsiveness ${ }^{3}$ recovery rates are better for those patients with severely traumatised but intact nerves. ${ }^{3,22}$

Taking these facts together, it can be stated that unilateral section of the chorda tympani goes mostly unnoticed from the gustatory and salivary point of view: the deficits are well compensated by the opposite side. In contrast, cutting the remaining chorda tympani may result not only in gustatory but also salivary symptoms.

\section{Conclusion}

The chorda tympani nerve carries not only taste but also parasympathetic fibres for the sublingual, submandibular and accessory salivary glands of the oral cavity. Cutting one chorda tympani does not lead to any salivary symptoms, whereas cutting both induces subjective and objectively measurable xerostomia. The present findings further emphasise the importance of preserving the chorda tympani nerve during middle-ear surgery whenever possible.

\section{Acknowledgements}

This study was supported by a grant from the Swiss National Fund for Scientific Research (SSMBS grant number PASMA-119579/1) to BNL.

\section{References}

1 Bull TR. Taste and the chorda tympani. J Laryngol Otol 1965;79:479-93

2 Landis BN, Beutner D, Frasnelli J, Huttenbrink KB, Hummel T. Gustatory function in chronic inflammatory middle ear diseases. Laryngoscope 2005;115:1124-7

3 Saito T, Manabe Y, Shibamori Y, Yamagishi T, Igawa H, Tokuriki M et al. Long-term follow-up results of electrogustometry and subjective taste disorder after middle ear surgery. Laryngoscope 2001;111:2064-70

4 Wolf O. Concerning the function of the chorda tympani [in German]. Zeitschrift für Ohrenheilkunde 1880:9:152-8

5 Mueller CA, Khatib S, Naka A, Temmel AF, Hummel T. Clinical assessment of gustatory function before and after middle ear surgery: a prospective study with a two-year follow-up period. Ann Otol Rhinol Laryngol 2008;117: 769-73

6 Mahendran S, Hogg R, Robinson JM. To divide or manipulate the chorda tympani in stapedotomy. Eur Arch Otorhinolaryngol 2005;262:482-7

7 Gopalan P, Kumar M, Gupta D, Phillipps JJ. A study of chorda tympani nerve injury and related symptoms following middle-ear surgery. J Laryngol Otol 2005;119:189-92

8 Michael P, Raut V. Chorda tympani injury: operative findings and postoperative symptoms. Otolaryngol Head Neck Surg 2007:136:978-81

9 Rice JC. The chorda tympani in stapedectomy. $J$ Laryngol Otol 1963;77:943-4

10 House HP. Early and late complications of stapes surgery. Arch Otolaryngol 1963;78:606-13

11 Landis BN, Welge-Luessen A, Bramerson A, Bende M, Mueller CA, Nordin S et al. "Taste Strips" - a rapid, lateralized, gustatory bedside identification test based on impregnated filter papers. J Neurol 2009;256:242-8

12 Osterberg T, Landahl S, Hedegard B. Salivary flow, saliva, $\mathrm{pH}$ and buffering capacity in 70-year-old men and women. Correlation to dental health, dryness in the mouth, disease and drug treatment. J Oral Rehabil 1984;11:157-70

13 Becks H, Wainwright WW. Human saliva: XIII. Rate of flow of resting saliva of healthy individuals. $J$ Dent Res 1943;22:391-6

14 Chen JM, Bodmer D, Khetani JD, Lin VV. Tactile dysgeusia: a new clinical observation of middle ear and skull base surgery. Laryngoscope 2008;118:99-103

15 Toremalm NG, Bjerre I. Surgical elimination of drooling. Laryngoscope 1976;86:104-12

16 Grant R, Miller S, Simpson D, Lamey PJ, Bone I. The effect of chorda tympani section on ipsilateral and contralateral salivary secretion and taste in man. J Neurol Neurosurg Psychiatry 1989:52:1058-62

17 Saito T, Shibamori Y, Manabe Y, Yamagishi T, Igawa H, Yamamoto T et al. Intraoperative identification of regenerated chorda tympani nerve and its relationship to recovered taste function. ORL J Otorhinolaryngol Relat Spec 2001;63: 359-65

18 Saito T, Shibamori Y, Manabe Y, Yamagishi T, Yamamoto $\mathrm{T}$, Ohtsubo T et al. Morphological and functional study of regenerated chorda tympani nerves in humans. Ann Otol Rhinol Laryngol 2000;109:703-9

19 Saito T, Shibamori Y, Manabe Y, Yamagishi T, Igawa H, Ohtsubo $\mathrm{T}$ et al. Incidence of regeneration of the chorda tympani nerve after middle ear surgery. Ann Otol Rhinol Laryngol 2002;111:357-63

20 Just T, Homoth J, Graumuller S, Pau HW. Disorders and recovery of taste function after middle ear surgery [in German]. Laryngorhinootologie 2003;82:494-500 
21 Berteretche MV, Eloit C, Dumas H, Talmain G, Herman P, Tran Ba Huy P et al. Taste deficits after middle ear surgery for otosclerosis: taste somatosensory interactions. Eur J Oral Sci 2008;116:394-404

22 Sakagami M, Sone M, Tsuji K, Fukazawa K, Mishiro Y. Rate of recovery of taste function after preservation of chorda tympani nerve in middle ear surgery with special reference to type of disease. Ann Otol Rhinol Laryngol 2003;112:52-6

Address for correspondence:

Dr Basile N Landis,

Department of Otorhinolaryngology,
Geneva Neuroscience Center,

University of Geneva Medical School,

Rue Gabrielle-Perret-Gentil 4,

CH-1211 Geneva, Switzerland.

Fax: +4122 3728240

E-mail: bnlandis@yahoo.co.uk

Dr B N Landis takes responsibility for the integrity of the content of the paper.

Competing interests: None declared 